\title{
Challenges, solutions and lessons learnt in 7 years of Service Management at CERN
}

\author{
David Martín Clavo ${ }^{1, *}$, Barbara Brugger $^{2}$, Nicole Cremel $^{1}$, Catherine Delamare ${ }^{1}$, Isabel \\ Fernandez Gonzalez ${ }^{2}$, Eric Lienard ${ }^{2}$, Reinoud Martens ${ }^{2}$, Mats Moller ${ }^{1}$, Wayne Salter ${ }^{1}$, \\ Zhechka Toteva ${ }^{1}$ and Olaf Van der Vossen ${ }^{2}$ \\ CERN, Esplanade des Particules 1, 1211 Geneva, Switzerland, ${ }^{1}$ IT Department, ${ }^{2}$ SMB Department
}

\begin{abstract}
CERN has been using ITIL Service Management methodologies and ServiceNow since early 2011. Initially a joint project between just the Information Technology and the General Services Departments, now most of CERN is using this common methodology and tool, and all departments are represented totally or partially in the CERN Service Catalogue. We introduce a summary of the current situation of Service Management at CERN, as well as its recent evolution. We discuss service onboarding, user experience, and tool configuration, outlining the challenges faced, solutions adopted and some important lessons learnt. Some of the most important points relate to CERN's common process and service catalogue, which have enabled scalability; hiding complexity from users in order to improve user experience; and the good practices to configure CERN's Service Management application which have been learnt in the 8 years of the project. Finally, we present ongoing and future work, such as the upgrade of the CERN Service Portal and bringing even more new services board.
\end{abstract}

\section{Introduction}

With the planned start of the LHC in 2008, the number of users at CERN started to grow at a very quick pace, while the number of CERN Staff decreased. Therefore, CERN services needed to do "more with less" and have been thinking of applying Service Management methodologies since then.

February 2011 was the go-live of the CERN Service Management tool, ServiceNow, and the Service Desk, as a joint Service Management project between the General Services (GS) and Information Technology (IT) departments [1]. Since then, the number of tickets created by users every year has been multiplied by 5 between 2011 and 2018. The number of users has also grown many times: there are now almost 2000 supporters, compared to 400 in 2011 , and around 20000 unique callers.

There are signs of positive maturement and increased quality of CERN services, such as the fact that currently more requests are created than incidents daily, and the fact that the number of created incidents is going down, especially if we only consider human-generated incidents. This growth and maturement is illustrated in the charts present in Figure 1.

\footnotetext{
* Corresponding author: david.martin.clavo@cern.ch
} 

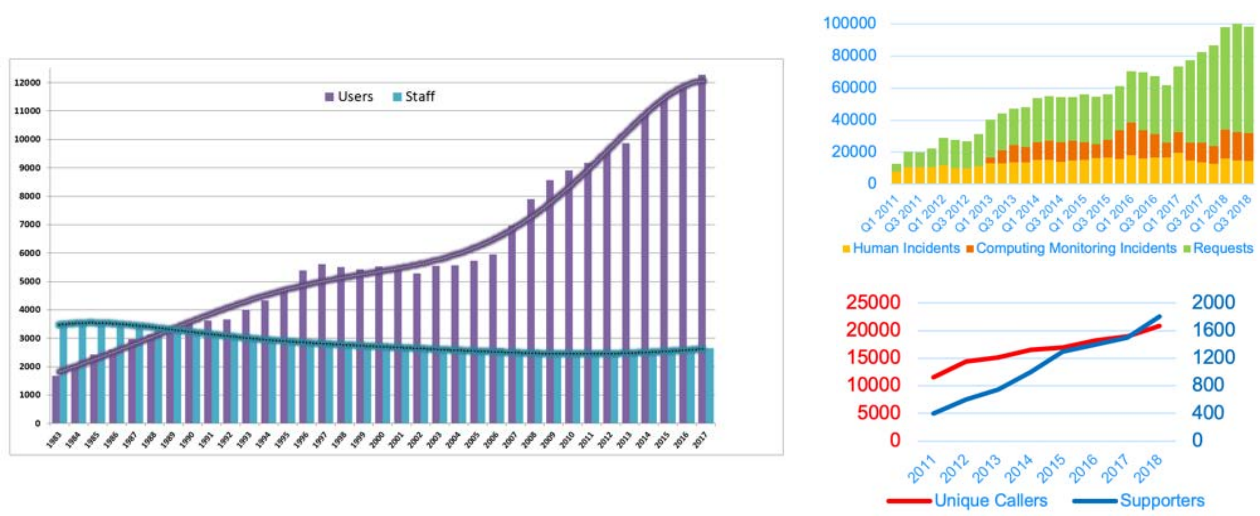

Fig. 1. CERN Users vs Staff evolution, and growth of Service Management at CERN

In this paper, we introduce first the challenges, solutions and lessons learnt about bringing services on board of the Service Management project, related to the CERN Service Catalogue, the Service Management processes, and contract management. Then, we present the topic of User Experience, looking at several parts of the project where it was improved or we learnt important lessons. After that, we will detail important lessons learnt with regard to configuring the Service Management tools used at CERN. We will conclude with a summary of the most important lessons learnt and of the present and future challenges.

\section{Bringing services on board}

\subsection{Service Catalogue}

The different services offered by CERN to its users are organised in the CERN Service Catalogue. This catalogue is two-dimensional: it splits the "What" from the "How". The "What" is represented by Service Elements and the "How" by Functional Elements.

At the beginning of the Service Management project in 2010, the challenge was to convince service managers of the value of having this kind of catalogue. Communication in the form of several presentations and meetings was given, and the Service Catalogue started to be built even before the selection of the supporting computer tool. A "big bang" approach was adopted: onboarding more than 400 Service Elements and Functional Elements into the project's initial go-live. This was due especially to IT requirements, as there was a deadline to replace the previous tool, BMC Remedy [2]. The service catalogue underwent a big review two years later, after most IT and GS services had already adopted the new methodology.

8 years later, at the end of 2018 , as the time constraints are not so severe, the CERN Service Management team prefers to take a step-by-step and hand-holding approach. When onboarding a new CERN group into the CERN Service Catalogue and Service Management methodologies, it is better to first implement a single use case; usually an internal one, or one where the migration is not disruptive. After the service users and supporters are convinced, the other services in the group will follow easily.

Continuous improvement and maintenance of the catalogue are critical. The service catalogue is not a one-time job; it needs to be maintained and have continuous improvement, to be up to date and adapt to changes in the organization. The total number of Service Elements and Functional Elements has grown from 400 in 2011 to more than 1000 at the end of 2018. After the IT and GS departments, the Administration sector (HR, Finance, etc.) also joined, and more recently many groups in the Accelerators sector as well. 


\subsection{Single process set}

As with the single Service Catalogue, CERN also has a single "Service Management Process set". This means that despite having different processes depending on the kind of ticket (Incident, Request, Work Task, etc.), these processes are the same across the different parts of the organization, such as IT, HR, facilities, engineering or finance.

The opposite is an "island" organization: different departments have different workflows or kinds of tickets, or even different tools. This introduces overhead, due to ticket conversions for example, and more maintenance.

A single and common process set enables scalability for onboarding new services: services are typically added to the service catalogue in half a day. The combination of the common two-dimensional service catalogue and process set, not only for IT but also for nonIT areas, attracts many other public and private organizations who come to visit CERN every year in order to understand this organisation method. Some of these organizations were struggling due to their "island" approach and moved to a more common process.

\subsection{Contract management}

Another service management activity is to manage contracts between the Organization and external companies that deliver a service, such as the Service Desk or the IT 2nd Line support. One of the challenges of this activity is to measure the service quality, the compliance and performance of the contractors. To perform these measurements, the CERN Service Management team learnt after several iterations that it is better to start with a few, simple Key Performance Indicators (KPIs), and follow through on using them. This is better than building and implementing a long list of KPIs, where only some are used in practice at all.

The three KPIs that have been adopted are: percentage of tickets with a dissatisfied user feedback; percentage of tickets with a breached Operational Level Agreement (OLA); and percentage of tickets with a non-compliance report. These KPIs have been incorporated into the contracts with the suppliers and were included in the call for tender documents. It is very important to think about what to measure while drafting a new contract: what really represents the quality of the service, but also what can be measured with the service management tool used. By including these KPIs in a contract, penalties or bonuses can be applied depending on the service level delivered by contractors.

The CERN Hotel service is one positive outcome example. A supplier company is responsible for the day-to-day operations. Since the last contract started, the Hotel has increased its revenues and reduced its charges, thanks to all work being tracked with service management tools, which encouraged the company to optimise the hotel management.

\section{User experience}

\subsection{Single process set}

Having a single process set for all departments, as described in section 2.2, is also beneficial for user experience. As Figure 2 illustrates, tickets will not be different whether they concern IT, HR, or other services; and they will also not be different whether they are created by users calling the Service Desk, using the Service Portal website [3], or sending an email.

This also enables scalability in the number of users that can use the system, as users do not need to learn new ways of contacting the services as more services are incorporated. Users have a single point of contact and not a different one per department. 


\begin{tabular}{|c|c|c|c|}
\hline \multicolumn{2}{|c|}{ Service Portal } & ServiceNow & Service Desk \\
\hline \multicolumn{4}{|c|}{ Service Management } \\
\hline IT & Facilities & HR & Finance \\
\hline
\end{tabular}

Fig. 2. Common Service Catalogue and single process set across CERN, and single point of contact

One remaining challenge is that the usage of email is still important, though decreasing, as shown in Figure 3. In the second half of 2018, 36\% of the tickets were created by email, versus $46 \%$ from the Service Portal, $8 \%$ by phone, $8 \%$ from ServiceNow and $2 \%$ by walkin. While these figures are better than in 2012 , where only $25 \%$ of the tickets were created form the web versus $50 \%$ by email and $25 \%$ by phone, there is still a lot of room for improvement, as tickets created by online forms generate less work both for the user and the supporter, compared to "free text" tickets created by email.

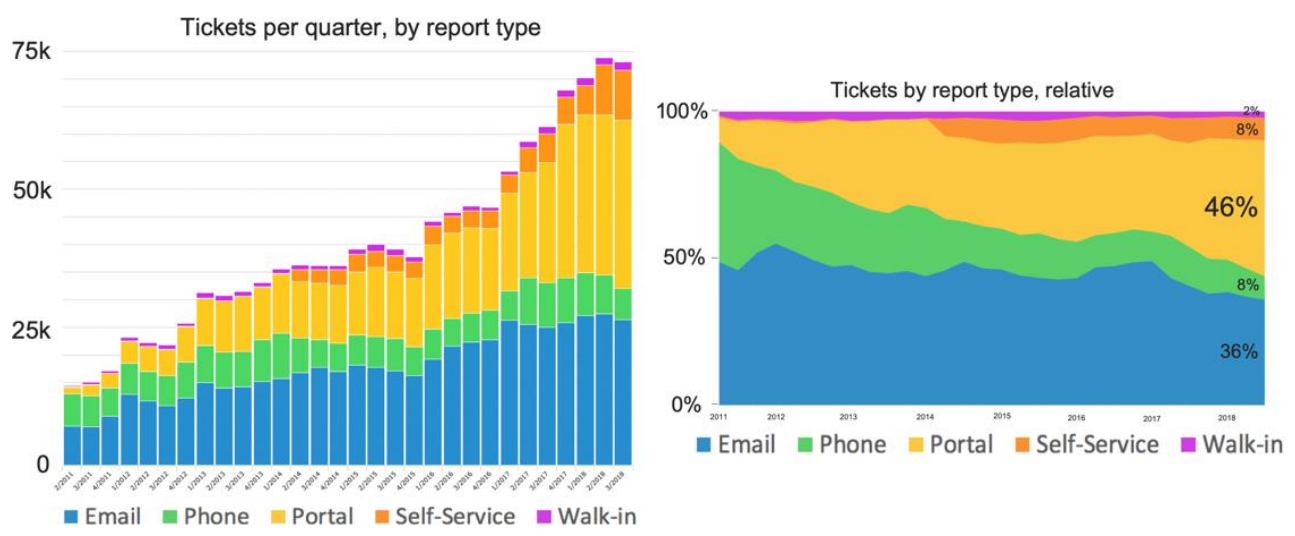

Fig. 3. Evolution of tickets at CERN by report type. Percentages on the right correspond to Q3 2018.

\subsection{Incident vs Request}

CERN has a single service management process set across departments. Nevertheless, inside this process set, several processes for different types of tasks exist. Incident Management and Request Management are the two most important ones. These two different processes need to be different: for example, a Request may need approval from a supervisor to be granted, but an Incident does not need an approval to be resolved. The Service Level Agreements (SLAs) for these two are different as well: generally, an incident needs to be resolved faster than a request.

To implement this difference, two different kinds of tickets were used in ServiceNow, with different identifiers, "INCxxxxxxx" and "RQFxxxxxxx", and different tables underneath. This can lead to a lot of confusion for users. For example, when a user sends an email to the Service Desk, the system creates an incident ticket by default; but very often the Service Desk agents convert the incident into a Request with a different identifier.

At the beginning of the Service Management project at CERN, it would have been possible, with significant work, to merge both kinds of tickets and use a unique identifier, "Txxxxxx", with only a "Ticket Type" attribute indicating its kind. This would avoid the need for ticket conversions, therefore hiding this complexity from end users, while still enabling different processes. However, due to many integrations between ServiceNow and other systems, and business logic built around this separation, it became impossible later. 


\subsection{Other processes}

Other service management processes implemented by the Service Management project include Service Level Management, Change Management and Problem Management. However, these processes are only used with various degrees of adoption.

Measurement of SLAs is active by default for all Incident and Request tickets since very early in the project. However, at the time many CERN services were not mature enough in their usage of service management methods and tools. As a result, due to inertia, many services ignore the SLA process. Recently, some services are starting to use it more, but adoption remains a challenge.

The Change Management process was designed as a "one size fits all" process, with many possible stages for a change: admission, evaluation, approval, plan \& build, test, deployment, review and closed. However, for many simple changes, these stages proved too many, and the UI of the tool cannot currently show different stage sets depending on the change type. Due to this complexity, this process has only been adopted by a few services.

The lesson to build a simple UI was used for the Problem Management process. A Problem ticket has less possible states: assigned, waiting for change, known error and closed. It is currently used more than the Change Management process, though most services do not use it or use other tools. Fostering adoption of this process at CERN and showing its benefits to service managers remain challenges for the CERN Service Management team.

\subsection{Online forms}

From the beginning of the Service Management project, one challenge to tackle was to reduce the amount of back-and-forth communication between caller and supporter, i.e. avoid many messages with questions and answers for incidents and requests. The solution was to use lightweight forms in order to request up-front the required specific information from the user, depending on the type of support case. Every day, hundreds of tickets are created at CERN via these forms, which saves many hours of work compared to treating "free text" tickets.

This choice was possible thanks to the fast development time with the selected tool, ServiceNow: on average a lightweight form takes only between 1 and 5 days to build. Currently, more than 550 forms are visible in the Service Portal and in ServiceNow. Despite their relative simplicity, maintaining all these forms is a challenge, especially during upgrades of the underlying platform, ServiceNow. Another challenge, already mentioned in section 3.1, is the remaining use of email and phone to create tickets.

\subsection{Ticket feedback}

Another example of the benefit of simplification is the Ticket Feedback process. Originally, there was a complex feedback dialog which was not easy to open. A simpler dialog, with "smileys", was implemented in 2015. These "smileys" also appear in the email notification that users receive when a ticket is resolved. Immediately after this simplification, 10 times more users started to give feedback on tickets, with currently $11 \%$ of users giving feedback across all CERN services. More feedback from users also results in the continuous improvement of documentation, procedures, and communication. The old and new feedback dialogs are shown in Figure 4.

Nevertheless, a remaining challenge to solve is that while service supporters now understand better what users think about their ticket handling, they do not always know their opinion about the quality of the service itself. Ways of measuring this are being discussed. 

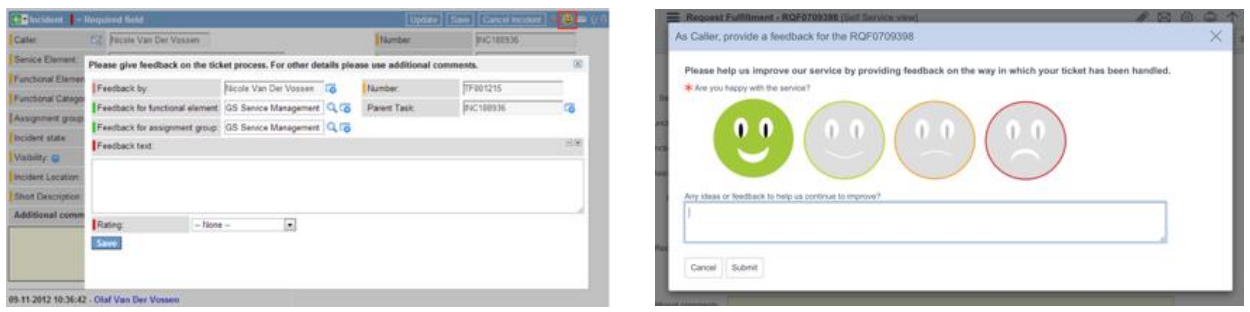

Fig. 4. Old (left) and new (right) ticket feedback dialogs

\subsection{Knowledge base}

The CERN Knowledge Base is a collection of Articles with useful information for end users and supporters, available in the Service Portal and in ServiceNow. In 2015, the process to publish and review an article was simplified. Before, users had to first create "Submissions" that were then published into "Articles". Submissions had to be created in order to edit KB Articles as well. After the simplification, Submissions are no longer needed, and the publishing and review workflow uses instead a "state" attribute of the KB Article.

As a result, creating and keeping up to date the Knowledge Base became simpler, resulting in an increase of the quality of the information available for users.

Nevertheless, many CERN users are not aware of the Knowledge Base. The current Service Portal does not promote it in its homepage, and does not try to propose KB Articles to users while they are creating tickets. The new version of the Service Portal, in development at the end of 2018, will try to improve this situation, which should result in more tickets being intercepted before being created thanks to users reading related KB Articles.

\subsection{Service Status Board and Service Availability Overview}

The Service Status Board [4] and the Service Availability Overview [5] are two websites built on top of the CERN Service Portal. They bring transparency about service status to the end users. The Service Status Board (SSB) focuses on high level service status, with announcements created manually by supporters, while the Service Availability Overview (SAO) focuses on technical service status, with service levels reported by automated monitoring systems.

Both websites existed in another form even before the start of the CERN Service Management project in 2010, but are now more visible and integrated with the Service Catalogue. They are both considered important parts of the success of the project.

In both cases, the lesson learnt is again about the benefits of simplification. The Service Availability Overview now displays status as "Available", "Degraded" or "Unavailable" instead of a numerical value between 0 and 100. The Service Status Board is also undergoing a simplification of the different available sections.

Nevertheless, some users are still not aware of the SSB and the SAO. Increasing the user awareness of these two websites would reduce the number of tickets created during major service incidents or interventions. The new Service Portal will show ongoing service incidents and interventions to users creating tickets for a service. 


\section{Tool configuration}

\subsection{Customise vs Extend vs Out of the box}

CERN uses a computer tool for Service Management: maintaining and displaying the Service Catalogue, tracking all tickets, holding the Knowledge Base and much more. In the case of CERN and many other organizations, this tool is ServiceNow [6], which is a commercial product [7] with the characteristics of both the SaaS (Software as a Service) and PaaS (Platform as a Service) models.

When adopting a commercial tool, the first decision to take is either using it as it is, often called using it "Out of the Box", or modifying it to suit better the organization's needs. When deciding to configure it, there are two strategies: customising or extending.

- When using a tool "Out of the Box", it is not needed to build and maintain any new functionality. Upgrades are easier than when the tool has been modified. However, it is likely that many users and supporters will be unsatisfied, and opportunities to gain value or cut costs in their processes can be lost.

- "Customising" means to modify what is provided out of the box. This provides some head start, but upgrades to the system are troublesome. The provider of the tool might also modify the same functionality, breaking customisations and causing conflicts.

- The last strategy is "extending", which means to build new functionality "on the side", only when needed, instead of modifying existing functionality provided out of the box. This results in much easier upgrades than with the customisation strategy.

At CERN, adapting ServiceNow to use cases of its users and supporters was needed. After many years, the lesson learnt is that it is much better to extend than to customise.

An example of extension would be the CERN Service Portal website, or the forms that have been created to support service managing Data Privacy Protection, such as the Record of Processing Operations form.

An example of customisation that was done in the past was modifying the Work Task table and form. Work Tasks started as part of ServiceNow's Work Management module, which later evolved into Field Service Management. Each upgrade added buttons and logic which needed to be adapted every time, and required costly testing. For that reason, it would have been better to create functionality independent from the out of the box one.

\subsection{Integrations with other systems}

CERN's Service Management tool (ServiceNow) is integrated with more than 15 other CERN systems, in order to pull, push or synchronise information with them. Integrations allow to support service operation, increase value of the tool, and reduce support costs for the services.

For example, ServiceNow pulls core data from the CERN Foundation database; computer configuration information from LanDB, INFOR, PuppetDB, the IT CMDB and LayoutDB; pushes service catalogue information to several databases; and synchronises tickets with other systems such as GGUS, INFOR, JMT, JIRA and PLAN. A particularly interesting integration is the one with the monitoring system of the CERN Data Centre [8,9].

After implementing so many integrations, the CERN ServiceNow developer team has learnt than when possible, it is better to implement one-way integrations rather than two-way. Having one system read and write from another is more resilient to change than both systems reading and writing. In all cases, fluid communication between teams, as well as quick interteam support are critical. Maintenance of many integrations remains a challenge due to the different technical know-hows needed to maintain integrations of many different types. 


\section{Conclusions}

\subsection{Summary}

In this paper we have presented some of the challenges, solutions and lessons learnt about onboarding services into the Service Management project, user experience, and tool configuration, since the Service Management project started at CERN in 2010.

As shown in the "Bringing Services on Board" section, having a common process set and service catalogue across the organization enabled scalability in terms of services and users.

Also, as demonstrated through many examples in the "User Experience" section, simplification of processes and user interfaces is critical to ease adoption. This should always be kept in mind when designing new processes and interfaces.

Finally, in the "Tool Configuration" section, we have compared the different strategies to configure a Service Management computer tool. If the tool needs to be modified, which was the case at CERN, the best strategy to follow is to extend instead of customise.

\subsection{Most important current and future challenges}

In terms of onboarding services, the most important current challenge faced by the CERN Service Management team is the CERN Data Privacy Protection effort. New processes have been developed in order to support all CERN services, and the remaining CERN services not present in the Service Catalogue will very soon add their services in order to be able to edit and publish their Service Privacy Notices.

In terms of improving the user experience, a new Service Portal is currently being developed, with the goal of making the user interface more usable and reducing the number of tickets created by users. For this, the Knowledge Base and the Service Status Board will be more exposed to end users.

In the future, we will work on enhancing the Service Status Board with subscriptions; raising awareness of some processes, such as Problem Management; and finally, exploring the use of Machine Learning for assisting the supporters in ticket classification, routing and prioritisation.

\section{References}

1. Z. Toteva et al 2012. Service management at CERN with Service-Now. J. Phys.: Conf. Ser. 396062022

2. BMC Remedy website: http://www.bmc.com/it-solutions/remedy-itsm.html

3. CERN Service Portal: https://cern.ch/service-portal

4. CERN Service Status Board: https://cern.ch/ssb

5. CERN Service Availability Overview: https://cern.ch/sls

6. CERN's ServiceNow instance: https://cern.service-now.com

7. ServiceNow company website: https://www.servicenow.com

8. P. Mendez Lorenzo et al 2014. Migration of the CERN IT Data Centre Support System to ServiceNow. J. Phys.: Conf. Ser. 513062032

9. D. Martin Clavo et al 2018. IT Service Management at CERN: Data Centre and Service monitoring and status. CHEP 2018 (to be published) 